
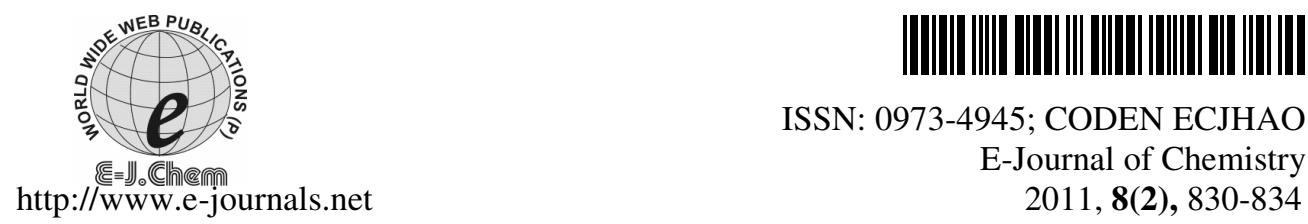

ISSN: 0973-4945; CODEN ECJHAO

E-Journal of Chemistry 2011, 8(2), 830-834

\title{
Antimycobacterial Activity of Some Synthesized Fluorinated Benzothiazolo Imidazole Compounds
}

\author{
B.S. SATHE* ${ }^{*}$ E. JAYCHANDRAN ${ }^{\S}$, \\ G.M SREENIVASA ${ }^{\S}$ and V. A. JAGTAP
}

Department of Pharmaceutical Analysis

Smt. S. S. Patil College of Pharmacy, Chopda - 425 107, India

${ }^{\S}$ P. G. Department of Pharmaceutical Chemistry

S. C. S. College of Pharmacy, Harapanahalli - 583 131, India

drbss1978@rediffmail.com

Received 18 July 2010; Accepted 5 September 2010

\begin{abstract}
Fluoro-3-chloroanilline treated with potassium thiocyanate in presence of glacial acetic acid and bromine was converted into 2-amino-6fluoro-7-chlorobenzothiazole, resulting into 2 -amino benzothiazole. The synthesized compound in presence of 2-phenyl-4-benzylidine-5-oxazolinone refluxed in pyridine to obtain 2-(2-phenyl-4-benzylidenyl-5-oxo-imidazolin -1-yl amino)-6-fluoro-7-substituted(1,3)benzothiazoles. The above said compound was treated with ortho, meta and para nitroanillines, ortho, meta, para chloroanillines, morpholino, piperazine, diphenylamine in the presence of DMF to obtain different derivatives. Some compounds showed promising antimicrobial activity.
\end{abstract}

Keywords: Flourine, Benzothiazole, Oxazalinone, Imidazoline, Antimycobacterial activity.

\section{Introduction}

Fluorobenzothiazoles and imidazoles exhibit the broad range of antibacterial ${ }^{1}$, antifungal ${ }^{2}$, anthelmintic ${ }^{3}$, anti-inflammatory ${ }^{4}$ and antitubercular ${ }^{5}$ activity. In the recent years, the chemistry of oxazolones ${ }^{6}$ has received much attention due to their use as intermediates for synthesis of some heterocyclic systems. In the present study we made an attempt to link $^{7-8}$ fluorobenzothiazoles with imidazoles for generating various derivatives, screened for antimycobacterial activity by using in-vitro models ${ }^{10}$. Benzylidine derivatives were found to possess MAO Inhibitory activity ${ }^{9}$, therefore in the present work we have treated oxazolones benzothiazole ring to get potent antimycobacterial leads. 


\section{Experimental}

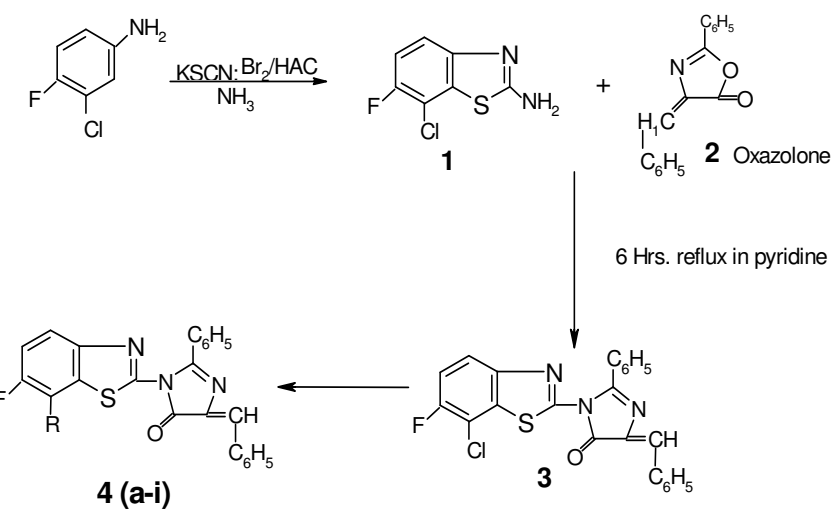

Purity of compounds was checked by TLC. Melting points were determined by open capillaries method and uncorrected. IR spectra $(\mathrm{NaCl})$ are recorded on FTIR (Schimadzu8300) spectrophotometer using nujol mull technique. For antimycobacterial activity in vitro by tube dilution technique using the human virulent $\mathrm{H}_{37} \mathrm{RV}$ strains of $\mathrm{M}$. tuberculosis.

\section{General Procedure}

\section{2-Amino-6-fluoro-7-chloro-(1,3)benzothiazole (1)}

To the glacial acetic acid $(20 \mathrm{~mL})$ which is cooled below room temperature, $8 \mathrm{~g}(0.08 \mathrm{~mol})$ of potassium thiocyanate and $1.45 \mathrm{~g}(0.01 \mathrm{~mol})$ of fluorochloroaniline was added. The mixture was placed in freezing mixture of ice and salt, mechanically stirred while $1.6 \mathrm{~mL}$ of bromine in $6 \mathrm{~mL}$ of glacial acetic acid was added, from a dropping funnel at such a rate that the temperature never rose beyond room temperature. After all the bromine was added (105 $\mathrm{min}$ ), the solution was stirred for $2 \mathrm{~h}$ below room temperature and at room temperature for $10 \mathrm{~h}$, it was then allowed to stand over night, during which period an orange precipitate settle at the bottom, water $(6 \mathrm{~mL})$ was added quickly and slurry was heated at $85{ }^{\circ} \mathrm{C}$ on a steam bath and filtered hot. The orange residue was placed in a reaction flask and treated with $10 \mathrm{~mL}$ of glacial acetic acid heated again to $85{ }^{\circ} \mathrm{C}$ and filtered hot. The combined filtrate was cooled and neutralized with concentrated ammonia solution to $\mathrm{pH} 6$. A dark yellow precipitate was collected. Recrystallised from benzene-ethanol mixture (1:1) after treatment with animal charcoal gave yellow plates of 2-amino-6-fluoro-7-chloro-(1,3) benzothiazole. After drying in an oven at $80{ }^{\circ} \mathrm{C}$, the dry material $(1 \mathrm{~g} 51.02 \%)$ melted at 210 $212{ }^{\circ} \mathrm{C}$. UV 307.4, $269 \mathrm{~nm}$, IR $1542 \mathrm{~cm}^{-1}$ (aromatic C=C) and $3475 \mathrm{~cm}^{-1}\left(\mathrm{NH}_{2}\right) ; 1456 \mathrm{~cm}^{-1}$ (thiazole), $1215 \mathrm{~cm}^{-1}$ (aromatic-F), $712 \mathrm{~cm}^{-1}$ (aromatic-Cl).

\section{2-Phenyl-4-benzylidine-5-oxazol-5-one (oxazolone) (2)}

Redistilled benzaldehyde was treated with benzoyl glycine (Hippuric acid) in presence of acetic anhydride (dry acetic acid) and anhydrous sodium acetate to get 4-benzylidene-2phenyl-oxazol-5-one(oxazolone). Upon washing with ice cold alcohol and then with boiling water (Yield 80\%), melted at $165-166{ }^{\circ} \mathrm{C}$, IR $(\mathrm{NaCl}) 1790 \mathrm{~cm}^{-1}$ (Lactone carbonyl) and another bond at $1650 \mathrm{~cm}^{-1}(\mathrm{C}=\mathrm{N}$ stretching).

2[2'- Phenyl -4'- benzidinyl-5'- oxo-imidazoline- 1yl- amino] -6 fluoro- 7- chloro $(1,3)$ benzothiazoles $(3)$

A mixture of 0.01 mol. of 2-amino-6-fluoro-7-chloro-(1,3)benzothiazole (1) with 2-phenyl4-benzylidine-5-oxazol-5-one (oxazolone)(2) refluxed in pyridine for 6-8 hours. Excess of 
pyridine was distilled off and resulting mass was poured on to crushed ice and neutralized with dil $\mathrm{HCl}$, filtered and product was recrystallised from ethanol. The dry material melted at $110-112{ }^{\circ} \mathrm{C}(72 \%) \cdot \mathrm{IR}(\mathrm{NaCl}) 3452 \mathrm{~cm}^{-1}$ (-NH stretching), $121 \mathrm{~cm}^{-1}(\mathrm{C}-\mathrm{F}), 677 \mathrm{~cm}^{-1}(\mathrm{C}-\mathrm{Cl}$ stretching), $3091 \mathrm{~cm}^{-1}$ ( $\mathrm{C}=\mathrm{C}$ stretching), $1601 \mathrm{~cm}^{-1}$ ( $\mathrm{C}=\mathrm{O}$ stretching).

\section{Preparation of various derivatives (4a-i)}

2[2'- Phenyl -4'- benzidinyl- 5'- oxo- imidazoline- 1yl- amino] -6 fluoro- 7- chloro $(1,3)$ benzothiazole (3) was treated with various aromatic amines. Refluxed for $2 \mathrm{~h}$. in presence of DMF (dimethyl formamide) yields various 2[2'- phenyl -4'- benzidinyl- 5'- oxoimidazoline- 1yl- amino] -6 fluoro- 7 - chloro $(1,3)$ benzothiazole derivatives $(\mathbf{4 a - i})$. The solid separated was cooled and poured on to crushed ice. The solid separated was filtered off, dried and recrystallised from benzene and super dry alcohol $(1 ; 1)$.

Table 1. Analytical data of the synthesized compounds (4a-i)

\begin{tabular}{|c|c|c|c|c|c|c|c|c|}
\hline \multirow{2}{*}{$\begin{array}{c}\text { Comp } \\
\text { No }\end{array}$} & \multirow{2}{*}{$\mathrm{R}$} & \multirow{2}{*}{$\begin{array}{c}\text { M.P. } \\
{ }^{\circ} \mathrm{C}\end{array}$} & \multirow{2}{*}{$\begin{array}{c}\text { Yield } \\
\% \\
\end{array}$} & \multirow{2}{*}{ M. F. } & \multicolumn{4}{|c|}{ Elemental Analysis \% } \\
\hline & & & & & & C & $\mathrm{H}$ & $\mathrm{N}$ \\
\hline \multirow{2}{*}{$4 \mathbf{a}$} & & \multirow{2}{*}{117} & \multirow{2}{*}{80} & \multirow{2}{*}{$\mathrm{C}_{27} \mathrm{H}_{17} \mathrm{~N}_{4} \mathrm{O}_{2} \mathrm{SF}$} & Found & 68.00 & 3.56 & 12.00 \\
\hline & & & & & Calc. & 67.50 & 3.54 & 11.66 \\
\hline \multirow{2}{*}{ 4b } & & \multirow{2}{*}{128} & \multirow{2}{*}{79} & \multirow{2}{*}{$\mathrm{C}_{27} \mathrm{H}_{18} \mathrm{~N}_{4} \mathrm{OSF}$} & Found & 70.01 & 3.90 & 12.13 \\
\hline & & & & & Calc. & 69.67 & 3.87 & 12.04 \\
\hline \multirow{3}{*}{$4 c$} & & \multirow{3}{*}{117} & \multirow{3}{*}{74} & \multirow{3}{*}{$\mathrm{C}_{35} \mathrm{H}_{23} \mathrm{~N}_{4} \mathrm{OSF}$} & Found & 74.90 & 4.15 & 10.09 \\
\hline & & & & & Calc. & 74.20 & 4.06 & 9.89 \\
\hline & & & & & Found & 65.09 & 3.56 & 13.89 \\
\hline \multirow[t]{2}{*}{ 4d } & & \multirow[t]{2}{*}{116} & \multirow[t]{2}{*}{66} & \multirow[t]{2}{*}{$\mathrm{C}_{29} \mathrm{H}_{18} \mathrm{~N}_{5} \mathrm{O}_{3} \mathrm{SF}$} & Calc. & 65.04 & 3.36 & 13.08 \\
\hline & & & & & Found & 66.00 & 3.89 & 14.02 \\
\hline \multirow[t]{2}{*}{$4 e$} & & \multirow[t]{2}{*}{126} & \multirow[t]{2}{*}{68} & \multirow[t]{2}{*}{$\mathrm{C}_{29} \mathrm{H}_{18} \mathrm{~N}_{5} \mathrm{O}_{3} \mathrm{SF}$} & Calc. & 65.04 & 3.36 & 13.08 \\
\hline & & & & & Found & 66.09 & 3.45 & 12.80 \\
\hline \multirow[t]{2}{*}{ 4f } & & \multirow[t]{2}{*}{122} & \multirow[t]{2}{*}{70} & \multirow[t]{2}{*}{$\mathrm{C}_{29} \mathrm{H}_{18} \mathrm{~N}_{5} \mathrm{O}_{3} \mathrm{SF}$} & Calc. & 65.04 & 3.36 & 13.08 \\
\hline & & & & & Found & 71.09 & 3.90 & 12.56 \\
\hline \multirow[t]{2}{*}{$4 g$} & & \multirow[t]{2}{*}{111} & \multirow[t]{2}{*}{72} & \multirow[t]{2}{*}{$\mathrm{C}_{29} \mathrm{H}_{19} \mathrm{~N}_{4} \mathrm{OSF}$} & Calc. & 71.02 & 3.87 & 11.42 \\
\hline & & & & & Found & 67.77 & 3.89 & 10.89 \\
\hline \multirow[t]{2}{*}{$4 h$} & & 85 & 77 & $\mathrm{C}_{29} \mathrm{H}_{18} \mathrm{~N}_{4} \mathrm{OSFCl}$ & Calc. & 66.28 & 3.42 & 10.66 \\
\hline & & & & & Found & 67.05 & 3.67 & 11.09 \\
\hline $4 \mathbf{i}$ & & 115 & 70 & $\mathrm{C}_{29} \mathrm{H}_{18} \mathrm{~N}_{4} \mathrm{OSFCl}$ & Calc. & 66.28 & 3.42 & 10.66 \\
\hline
\end{tabular}


Table 2. IR spectral data of the synthesized compounds (4a-i)

\begin{tabular}{cccccccc}
\hline $\begin{array}{c}\text { Comp. } \\
\text { Code }\end{array}$ & $\begin{array}{c}\mathrm{NH} \\
\mathrm{cm}^{-1}\end{array}$ & $\begin{array}{c}\text { Imidazoline ring } \\
\text { carbonyl cm }\end{array}$ & $\begin{array}{c}\mathrm{C}=\mathrm{N} \text { stretching } \\
\mathrm{cm}^{-1}\end{array}$ & $\begin{array}{c}\mathrm{C}=\mathrm{C} \text { stretching } \\
\mathrm{cm}^{-1}\end{array}$ & $\begin{array}{c}\mathrm{NO}_{2} \\
\mathrm{~cm}^{-1}\end{array}$ & $\begin{array}{c}\mathrm{C}-\mathrm{F} \\
\mathrm{cm}^{-1}\end{array}$ & $\begin{array}{c}\mathrm{C}-\mathrm{Cl} \\
\mathrm{cm}^{-1}\end{array}$ \\
\hline $\mathbf{4 a}$ & 3353 & 1640 & 1612 & 1485 & --- & 1167 & --- \\
$\mathbf{4 b}$ & 3200 & 1640 & 1673 & 1460 & --- & 1161 & --- \\
$\mathbf{4 c}$ & 3200 & 1630 & 1600 & 1490 & --- & 1163 & --- \\
$\mathbf{4 d}$ & 3300 & 1640 & 1600 & 1490 & 802 & 1167 & --- \\
$\mathbf{4 e}$ & 3350 & 1639 & 1613 & 1487 & 799 & 1163 & --- \\
$\mathbf{4 f}$ & 3400 & 1630 & 1640 & 1400 & 890 & 1167 & --- \\
$\mathbf{4 g}$ & 3351 & 1641 & 1610 & 1460 & --- & 1164 & --- \\
$\mathbf{4 h}$ & 3350 & 1643 & 1600 & 1462 & --- & 1169 & 714 \\
$\mathbf{4 i}$ & 3349 & 1637 & 1653 & 1493 & --- & 1160 & 714 \\
\hline
\end{tabular}

Screening for antimycobacterial activity (in vitro models)

Sterile Kirchner's medium was dispensed in each borosilicate test tube $(150 \times 20 \mathrm{~mm})$ and to this sterile horse serum $(0.5 \mathrm{~mL})$ was added. The stock solution was sterile by passing through a $0.2 \mathrm{~mm}$ polycarbonate sterile membrane (Nuclepore) filters. Further the serial dilution of test compounds were carried out. Test compounds at various concentrations (250, $125,62,32,16,8,4$ and $1 \mu \mathrm{g} / \mathrm{mL}$ ) were added to culture medium in a sterilized borosilicate test tube and strain of M.tuberculosis was inoculated at concentration (106 bacilli $/ \mathrm{mL})$. The tubes were incubated at $37^{\circ}$ for 21 days and then examined for the presence or absence of growth of the test organisms. All experiments were performed in triplicate. The lowest concentration, which showed no visible growth, was taken as the end point i.e. minimum inhibitory concentration (MIC). Rifampin and isoniazid (INH) were used as standard for antimycobacterial activity.

Table 3. Antimycobacterial activity of synthesized compounds (4a-i)

\begin{tabular}{ccc}
\hline Comp. No. & $\begin{array}{c}\text { Activity Data } \\
\text { Codes }\end{array}$ & $\begin{array}{c}\text { H37RV strain of } \\
\text { M.tuberculosis 21 days }\end{array}$ \\
\hline 01 & $\mathbf{4 a}$ & 20 \\
02 & $\mathbf{4 b}$ & 26 \\
03 & $\mathbf{4 c}$ & 25 \\
04 & $\mathbf{4 d}$ & 13 \\
05 & $\mathbf{4 e}$ & 15 \\
06 & $\mathbf{4 f}$ & 21 \\
07 & $\mathbf{4 g}$ & 17 \\
08 & $\mathbf{4 h}$ & 20 \\
09 & $\mathbf{4 i}$ & 17 \\
Standard 1 & Rifampicin & 0.25 \\
Standard 2 & Isoniazide & 0.007 \\
\hline
\end{tabular}

\section{Conclusion}

All the synthesized fluorinated benzothiazole imidazole compounds have given appreciable yield with satisfactory elemental analysis. It is inferred from the Table 3 that synthesized compounds (4a-i), have shown significant antimycobacterial activity. However, animal study and other studies are necessary for its activity and also there is a need to elucidate its mechanism of antimycobacterial action. 


\section{Acknowledgment}

The authors express thanks to Dr. A.S. Bobde, Haffkine Institute, Mumbai for providing testing facilities for Antimycobacterial activity.

\section{References}

1. Sangal S K and Rastivona P K, Chem Abstr., 1986, 104, 34029

2. Gurupadiaiah B M, Jaychandran E, Nargund L V G and Shivkumar B, Indian J Heterocycl., 1998, 7, 213-216

3. Labendeno N Yu, Chem Abstr., 1980, 92, 922882

4. Areas J J, Chem Abstr., 1991, 14, 71453

5. Jaychandran E, Nargund L V G, Shivkumar B and Kamal Bhatia, Oriental J Chem., 2003, 19(1), 139-142

6. Vogel A I, Vogel's Textbook of Practical Organic Chemistry; ELBS, 1978, 4, 884-885.

7. Merja B C, Joshi A M and Parikh K A, Oriental J Chem., 2003, 13(1), 157-160

8. Verma V M, Chturvedi A K and Pamar S S, J Pharm Sci., 1974, 463, 1740.

9. Bhusari K P, Khedkar P B, Umathe S N and Raghuramrao A, Indian J Heterocycl Chem., 2000, 13, 798-800

10. Shieke V G and Bodade A S, Chem Abstr., 1991, 11423845r 


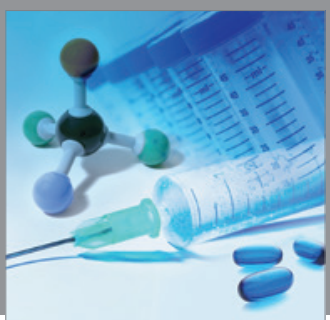

International Journal of

Medicinal Chemistry

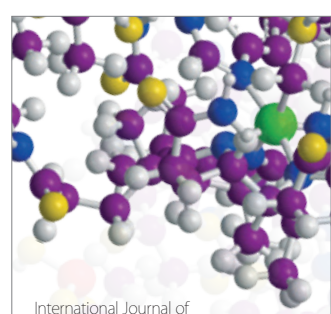

Carbohydrate Chemistry

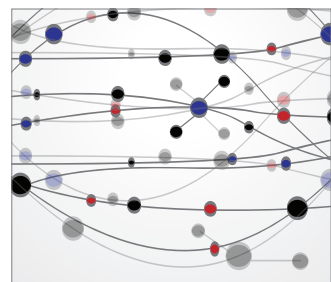

The Scientific World Journal
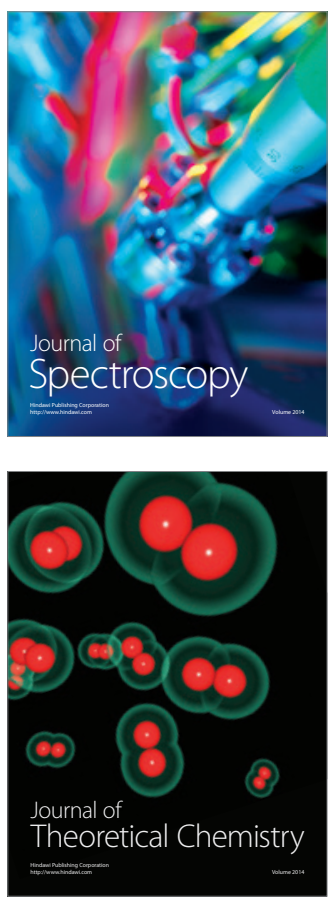
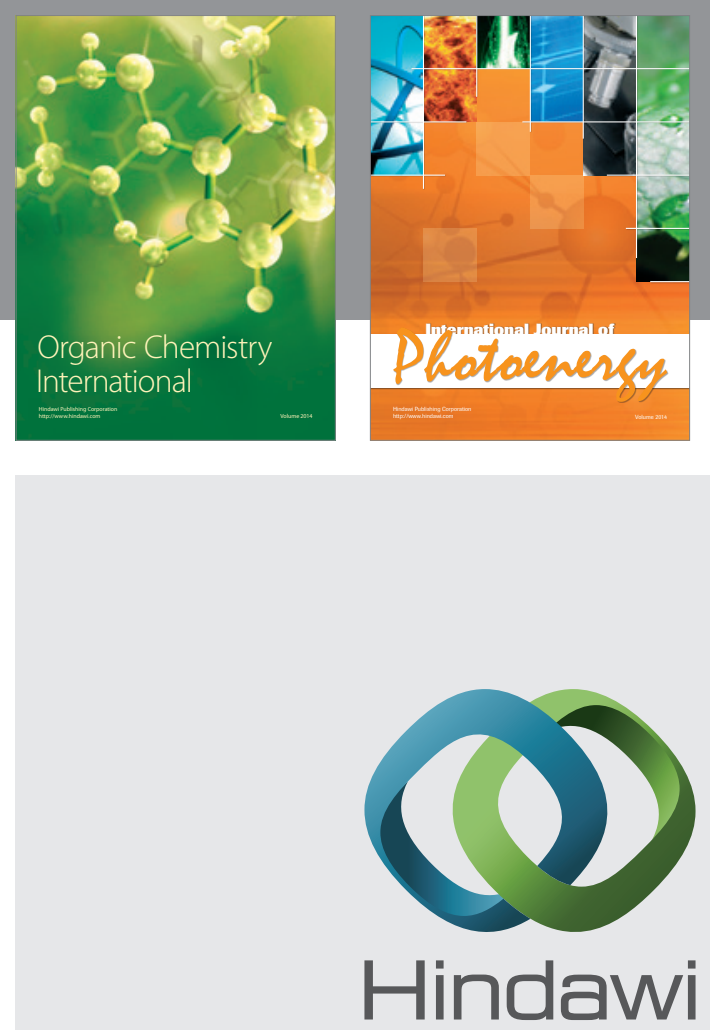

Submit your manuscripts at

http://www.hindawi.com
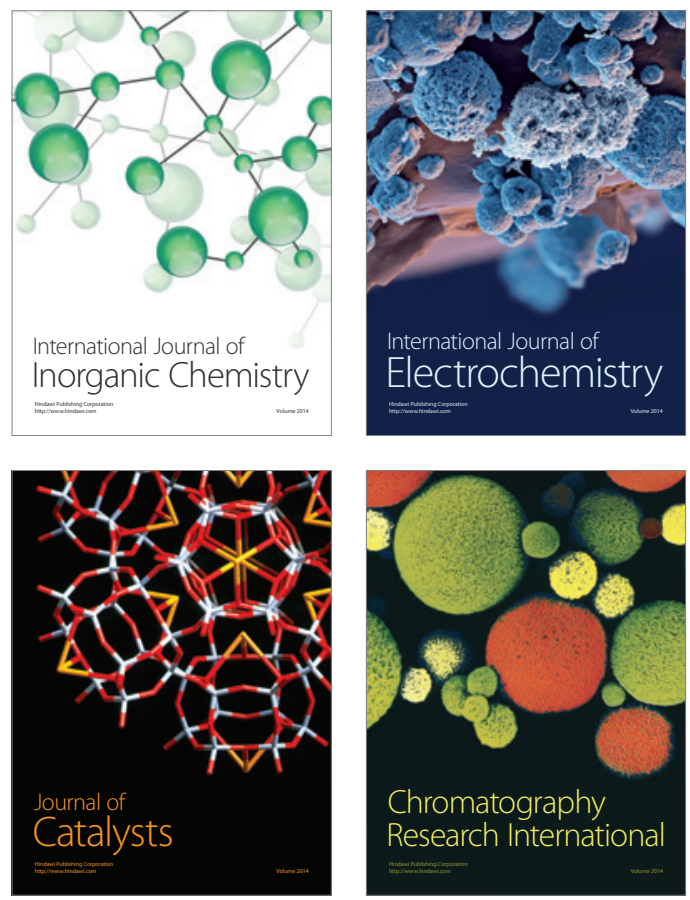
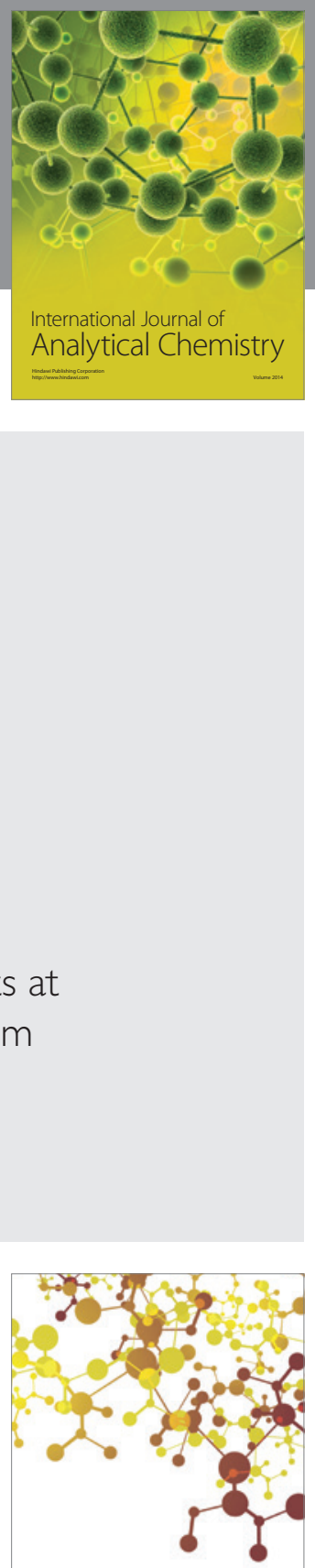

Journal of

Applied Chemistry
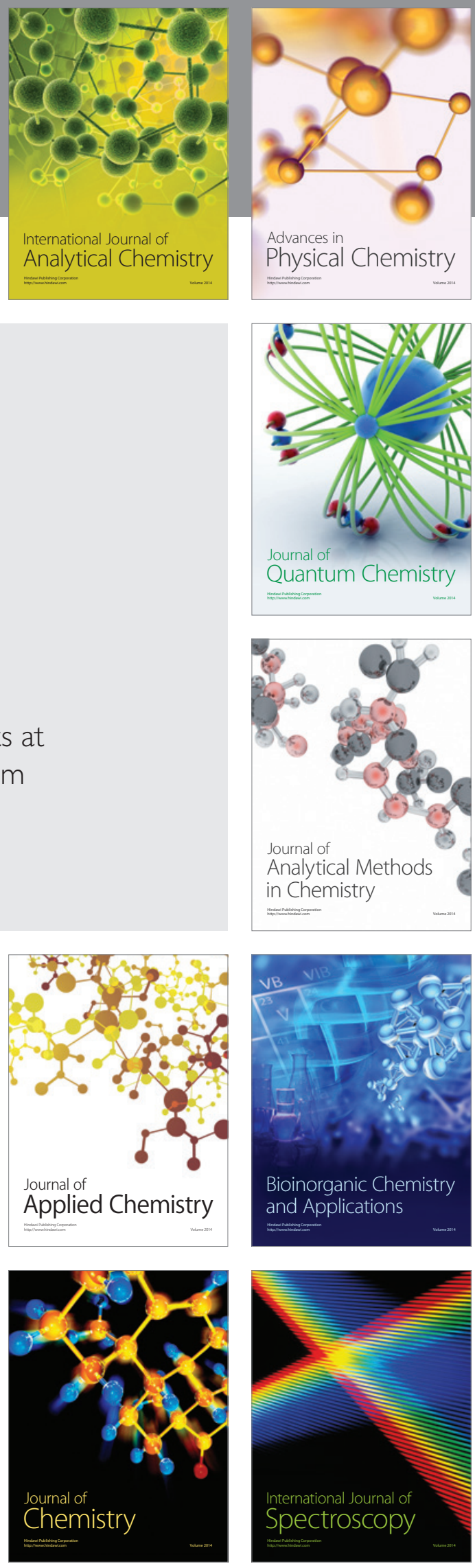